\title{
Es stinkt! Komponenten im Blütenduft von Orchidantha fimbriata
}

\author{
Martin Feulner, Marianne Lauerer \& Stefan Dötterl
}

\begin{abstract}
Orchidantha fimbriata Holtтum (Lowiaceae) flowered at the Ecological-Botanical Garden of the University of Bayreuth in December 2014. The zygomorphic flowers are notable for their conspicuous white-glossy lip (labellum) - but also for their strongly fetid odour. It was possible to collect scent from flowers and separate organs thereof. The scent was analysed by gas chromatography-mass spectrometry (GC-MS). The unpleasant odor was due to the three fetid compounds dimethyl disulfide, p-cresol and indole, all known to occur in plants, such as members of the aroid family and stapeliads, and serving as attractant for dung beetle and dung fly pollinators. The scent bouquet also contained a large number of sesquiterpenes and short-chain aliphatic esters. The latter substances are known as attractants for fruit beetles.
\end{abstract}

\section{Zusammenfassung}

Im Dezember 2014 blühte im Ökologisch-Botanischen Garten der Universität Bayreuth Orchidantha fimbriata HoltTum (Lowiaceae), deren bodennah stehende, große Blüten unangenehm, kotähnlich stinken. Dies war Anlass, Duftproben von einzelnen Blütenteilen (Labellum, Labellum plus Griffel-Staubblatt-Apparat, Sepalen) zu sammeln. Die Auswertung der Proben mittels Gaschromatographie gekoppelt mit Massenspektrometrie (GCMS) ergab, dass für die unangenehme Note des Duftes die Stoffe Dimethyldisulfid, p-Cresol sowie Indol verantwortlich sind. Diese Substanzen sind bereits aus anderen Kotoder Aasdüfte nachahmenden Pflanzenarten wie einigen Araceae und den Stapelien bekannt. Zudem enthielt das Duftbouquet neben einer großen Anzahl von Sesquiterpenen eine Reihe von Fettsäureestern, die dafür bekannt sind, Fruchtkäfer anzulocken.

\section{Taxonomie und Blütenbiologie von Orchidantha}

Orchidantha ist die einzige Gattung in der monotypischen Familie der Lowiaceen, die erst vor 3-19 Millionen Jahren entstanden ist (Stevens 2001). Sie ist damit die jüngste von acht Familien in der tropischen Ordnung der Zingiberales, zu der auch die Musaceae, Strelitziaceae, Heliconiaceae, Zingiberaceae, Costaceae, Cannaceae und Marantaceae gehören (STEvens 2011). Viele Vertreter der Zingiberales weisen Vogelbestäubung auf. So werden die Strelitzien und manche Ingwer-Arten von Nektarvögeln (Frost \& Frost 1981, Sakai et al. 1999) und Vertreter der Gattung Heliconia durch Kolibris bestäubt (STILES 1975).

Die Gattung Orchidantha enthält 16 beschriebene (Pedersen 2001, Jenjittikul \& Larsen 2002) und zumindest zwei unbeschriebene (Johansen 2005) Arten. Alle OrchidanthaArten sind in den Regenwäldern Südostasiens beheimatet (LARSEN 1961, 1973, 1993, NAGAmasu \& Sakai 1999, Pedersen 2001, JenjitTiKul \& Larsen 2002). Die meisten haben nur ein kleines Verbreitungsgebiet (Abb. 1) und sind selten. Die Arten sind krautig, ihre Blüten zwittrig, zygomorph und dreizählig. Sie ent- wickeln sich basal an der Pflanze und blühen nur etwa einen Tag lang (Johansen 2005). Die drei Kelchblätter sind zu einer langen, schlanken Röhre verwachsen, von den drei Kronblättern sind die beiden seitlichen sehr klein, während das mittlere, adaxiale zu einem großen Labellum umgebildet ist (Kress 1990). Dieses Labellum ist, u. a. auch neben der Struktur der Narbe (Pedersen \& Johansen 2004), eine Gemeinsamkeit der Gattung und hat ihr zu dem Namen Orchidantha verholfen. Dieser Name bedeutet „Orchideen-Blüte“ und verweist darauf, dass auch bei den Blüten der Orchideen ein Blütenblatt zu einem Labellum (Lippe) umgebildet ist. Pro Blüte sind bei Orchidantha fünf fertile Staubblätter mit kurzen Staubfäden vorhanden und drei synkarpe Fruchtblätter. Der Griffel endet in einer dreilappigen Narbe, die je nach Art in typischer Weise gezähnt oder gefranst ist.

Abb. 1: Geographische Verbreitung der einzelnen Arten der Gattung Orchidantha (verändert nach SAKAI \& InOuE 1999). Orchidantha fimbriata ist im Süden der Malaysischen Halbinsel beheimatet. Abkürzungen und Signaturen: $\mathrm{O}=$ Orchidantha; ? = die genaue Lokalität von $O$. borneensis auf Borneo ist unbekannt. 


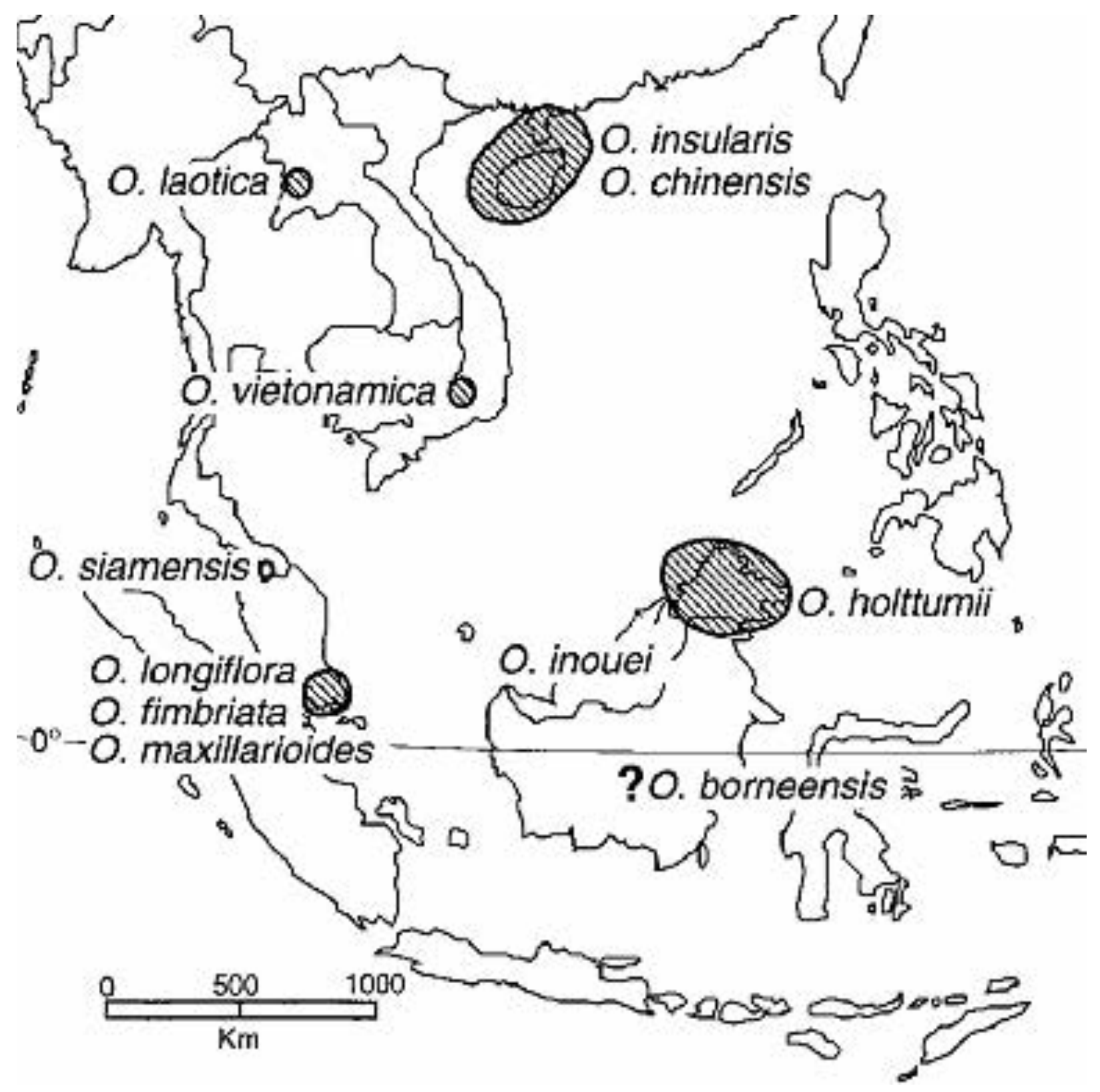

\section{Was ist bekannt über die Bestäubung} bei Orchidantha?

Pflanzen haben vielfältige, mutualistische Beziehungen zu Insekten entwickelt und belohnen ihre Bestäuber zum Beispiel mit Nektar, Pollen oder mit beidem. Aber dem ist nicht immer so. Sogenannte Täuschblumen, zu denen auch Orchidantha gehört (Pedersen \& JohanSEN 2004), täuschen den Bestäubern eine Belohnung vor, ohne diese zu haben (RENNER 2006). So ahmen beispielsweise die Blüten von Aasblumen den Duft von verfaulendem Eiweiß und oft auch die Farbe von vergammelndem Fleisch nach, um Aasfliegen oder -käfer anzulo- cken (Faegri \& van der Pijl 1979). Pflanzen wie verschiedene Stapelien ahmen Kotgeruch nach, wodurch Schmeißfliegen und andere Fliegengruppen angelockt werden (JürGENS et al. 2005). Ähnliches geschieht bei dem von $S_{A}$ KAI \& InOUe (1999) an der Art Orchidantha inoui beschriebenen Mistkäfer-Bestäubungssystem. Danach lockt die Blüte mit einem kotähnlichen Geruch Mistkäfer der Gattungen Onthophagus und Paragymnopleurus an. Die Käfer landen auf der großen, dunkelbraunen Lippe von Orchidantha inoui, in der Erwartung, einen Kothaufen als Nahrungsquelle und Brutsubstrat vorzufinden. Da Kothaufen sich 


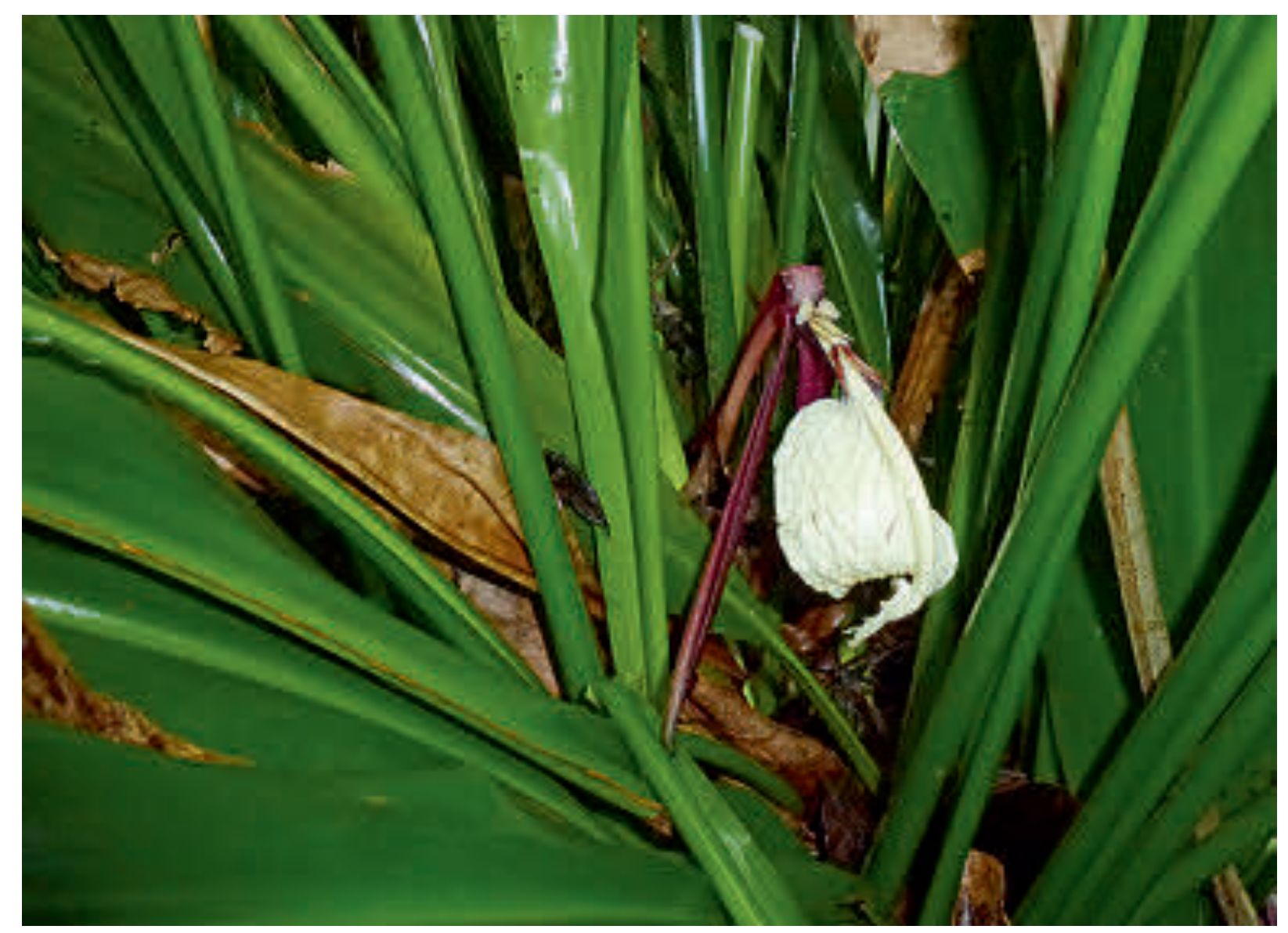

am Boden befinden, könnte damit auch die bodennahe Bildung der Blüten bei Orchidantha inoui (aber auch bei $O$. fimbriata) zusammenhängen. Die Dungkäfer suchen auf dem dunkelbraunen Labellum nach Kot, krabbeln schließlich unter den Narben-Staubblattapparat, streifen dabei den mitgebrachten Pollen an der Narbe ab und bestäuben die Blüte, ohne für sich eine Belohnung mitnehmen zu können. Um Selbstbestäubung zu vermeiden, wird der Griffel, der vor den Staubblättern liegt, nach oben gedrückt, wenn das Insekt tief in die Blüte kriecht, und bleibt auch beim Herauskriechen in dieser Position (SAKai \& InOue 1999).

Auch bei Orchidantha fimbriata liegt der Griffel vor den Staubblättern. Allerdings hat diese Art eine sogenannte „one-way“-Blüte, wobei der Bestäuber zunächst den Griffel passiert, dann unter den Antheren durchkriecht und Pollen aufnimmt und schließlich seitlich den Antheren-Griffelapparat wieder verlässt (Pedersen \& Johansen 2004). Basal und ventral der drei-lappigen Narbe befindet sich ein
Drüsengewebe, das sog. Viscidium. Beim Kriechen in die Blüte wird das Insekt dorsal mit dem Sekret aus dem Viscidium beschmiert, sodass der Pollen sehr gut am Tier kleben bleibt (Pedersen \& Johansen 2004).

Orchidantha fimbriata besitzt ein mit Sekret überzogenes, glänzendes, weißes Labellum und fällt wie $O$. inoui durch ihren unangenehmen, nach Kot riechenden Geruch auf. Wegen dieses Geruches vermutete HolTtum (1970) zunächst, dass Orchidantha fimbriata von Fliegen bestäubt wird, später wurde jedoch eine Bestäu-

Abb. 2 (oben): Die Blüte von Orchidantha fimbriata entwickelt sich an der Basis der krautigen Pflanze. Das Foto entstand im Tropenwaldhaus des ÖBG.

Abb. 3 (Seite 55, oben): Detail aus der Blüte von Orchidantha fimbriata mit den deutlich zu erkennenden beiden kurzen, zurückgerollten Petalen, den relativ kurzen Stamina und dem Griffel mit der charakteristisch 3-lappigen, fedrigen Narbe.

Abb. 4 (Seite 55, unten): Unterseite der Narbe von Orchidantha fimbriata. 


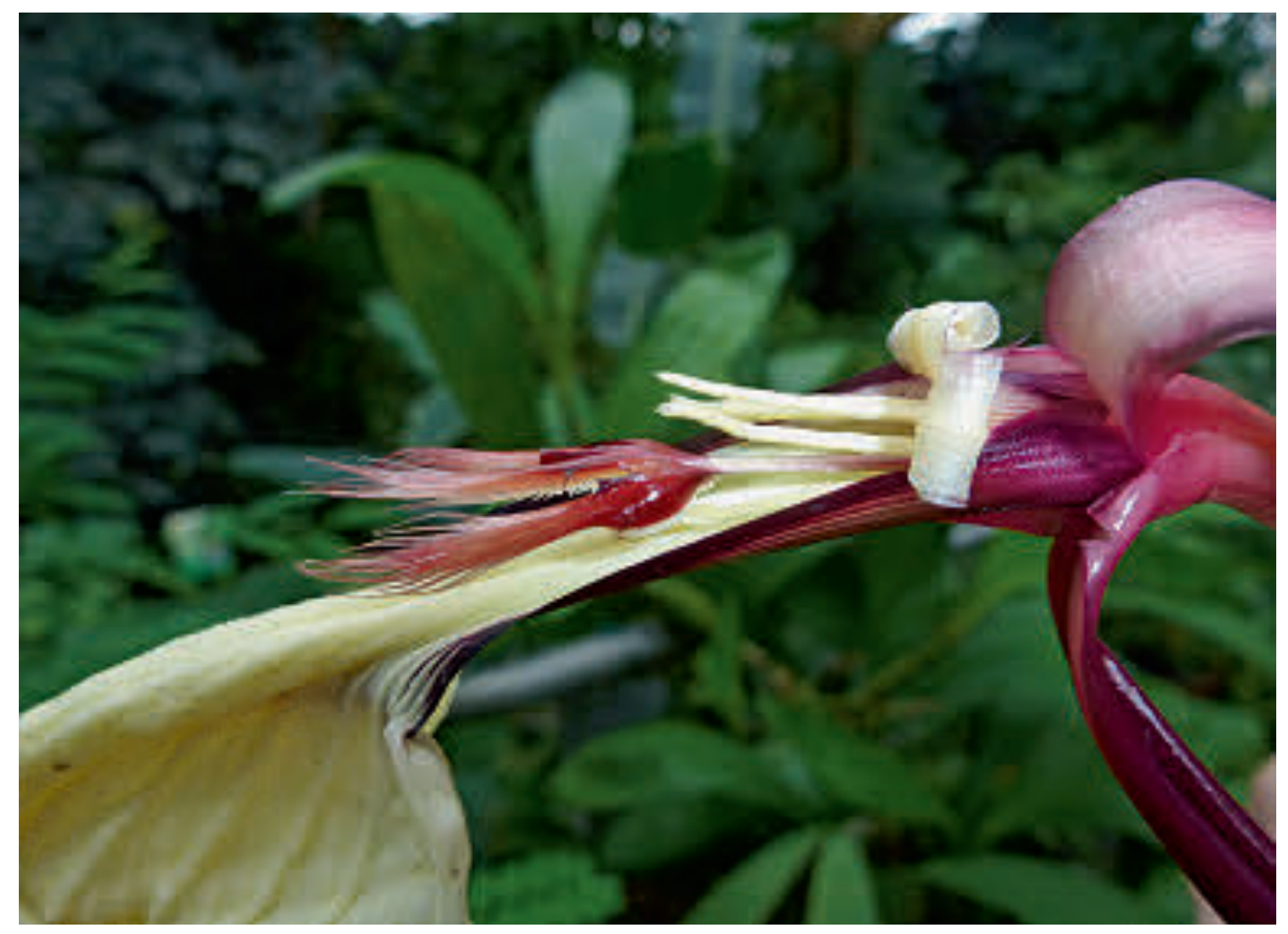

bung durch Fruchtkäfer beobachtet (Petersen $\&$ Johansen 2004). Detaillierte Untersuchungen zur Bestäubung von Orchidantha fimbriata fehlen aber bislang.

\section{Methode: Blütenduft sammeln und analysieren}

Im Tropenwaldhaus des Ökologisch-Botanischen Gartens der Universität Bayreuth (ÖBG) werden seit 2006 mehrere Individuen von Orchidantha fimbriata kultiviert, die vom Botanischen Garten Darmstadt stammen. Bei zwei Pflanzen öffneten sich zwischen dem 15. und 22. Dezember 2014 mehrere Blütenknospen. Der unangenehme Duft der auffälligen Blüten war Anlass, Gestank dieser Blüten zu sammeln und zu analysieren, um die abgegebenen Duftkomponenten zu identifizieren und evtl. Rückschlüsse auf mögliche Bestäuber ziehen zu können.

Mittels der Dynamischen Gasraum-Methode (Abb. 5) wurde an insgesamt vier Blüten von zwei Individuen von Orchidantha fimbriata

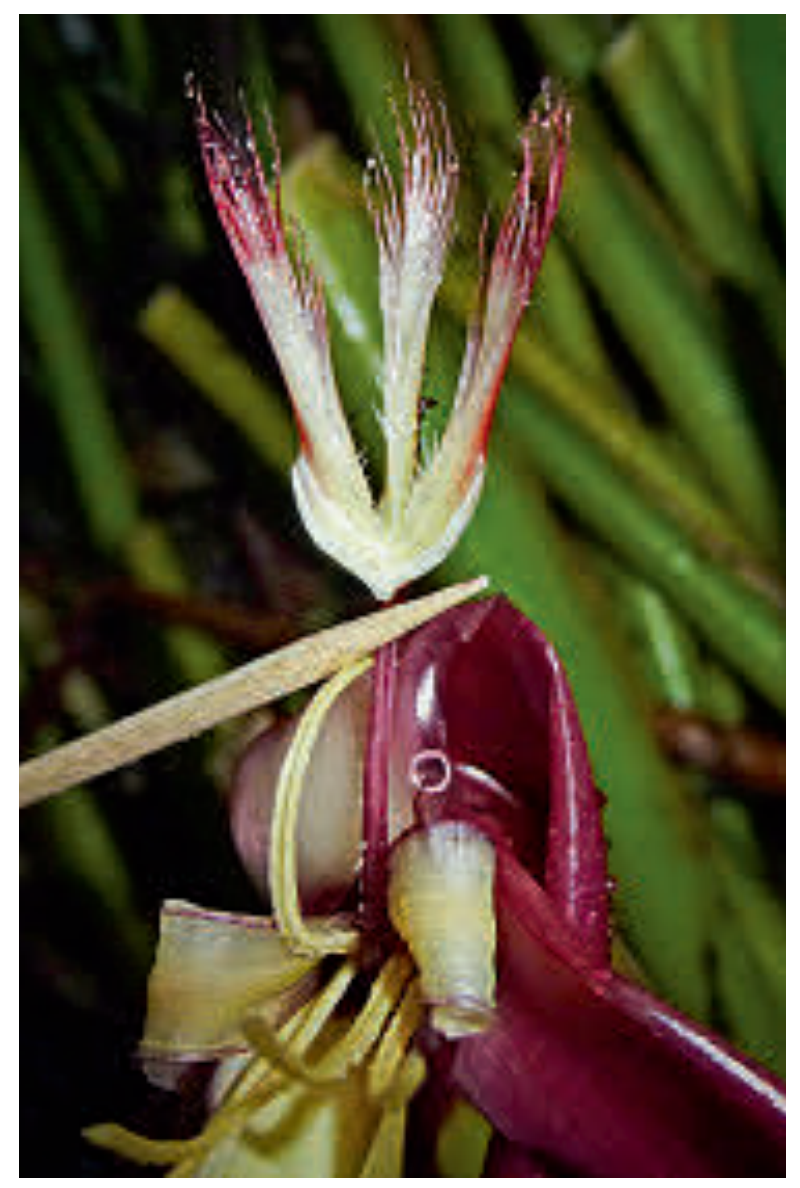




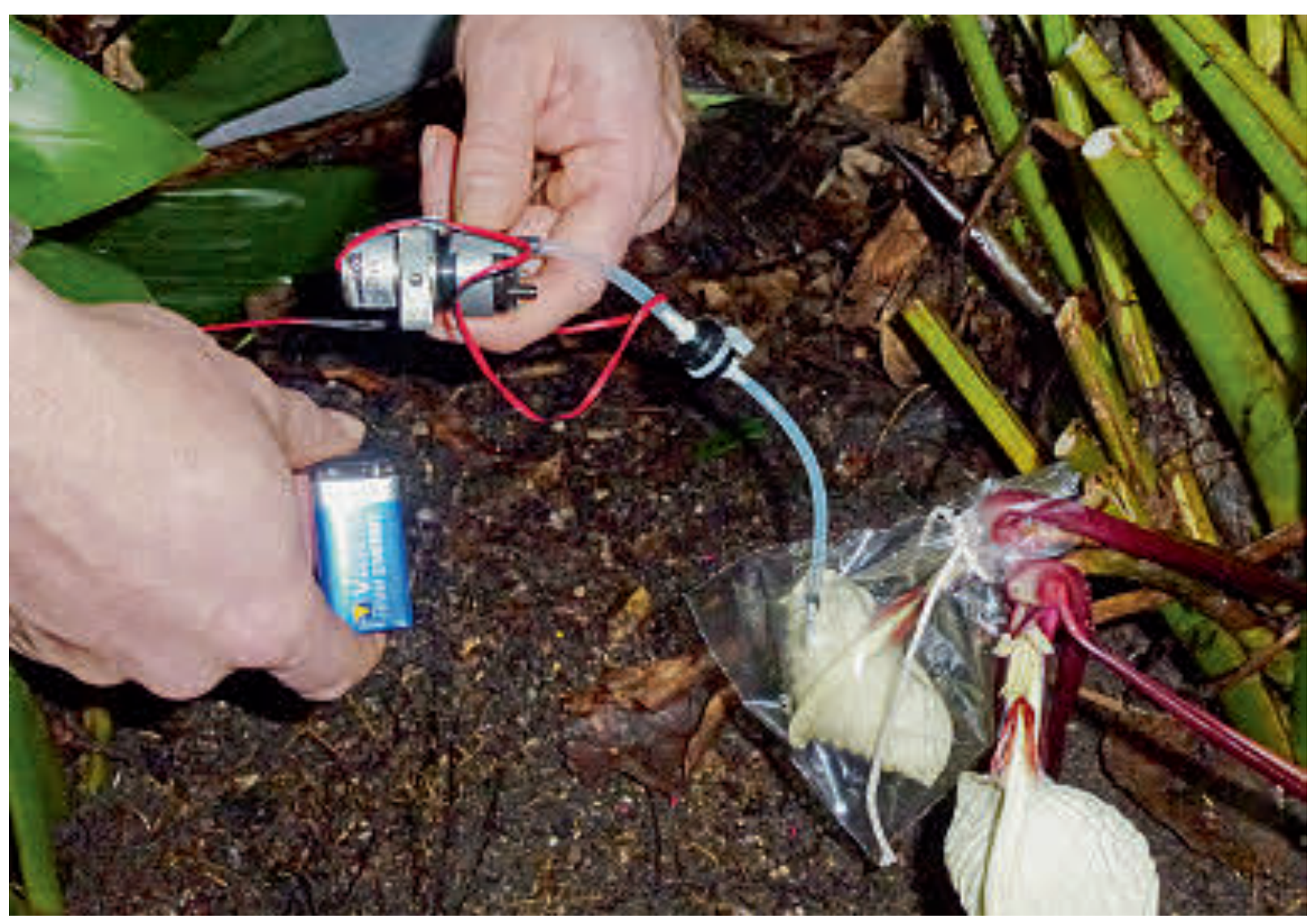

Duft abgesaugt. Die Blüte bzw. Teile der Blüte wurden dazu für 5 Minuten in eine Bratschlauch-Plastiktüte eingepackt. Anschließend wurde der Duft mittels einer Membranpumpe durch ein mit Duftstoff bindenden Stoffen gefülltes Glasröhrchen gesaugt. Der Duft wurde im Fachbereich Ökologie \& Evolution der Universität Salzburg mittels eines Gaschromatographen, gekoppelt mit einem Massenspektrometer, analysiert. An einer der beprobten Blüten wurden nacheinander unterschiedliche Blütenteile einzeln eingetütet und jeweils einmal der Duft genommen: vom Labellum, von den seitlichen Sepalen und vom Labellum zusammen mit dem Griffel-Antheren-Komplex.

\section{Ergebnisse der Duftstoffanalysen und Diskussion zur Bestäubung}

Schon beim Riechen an den unterschiedlichen Blütenteilen deutete sich an, dass nur das weiße Labellum den unangenehmen Geruch abgibt. Dies wurde durch die Analysen der Duftstoffe bestätigt. Die Sepalen waren weitgehend geruchlos. Die Duftbouquets der Proben des
Labellums alleine und vom Labellum zusammen mit dem Griffel-Antheren-Komplex unterschieden sich nicht. Dabei war der typische Kotgeruch auf die unangenehm riechenden Stoffe Dimethyldisulfid, p-Cresol sowie Indol zurückzuführen, wie man sie schon aus Untersuchungen an anderen Kot- oder Aasgeruch nachahmenden Pflanzen kennt (Jürgens et al. 2006). Dabei war Dimethyldisulfid in geringer Menge und die anderen beiden Stoffe etwa zu gleichen Teilen und höher konzentriert im Duft enthalten. Neben diesen typischen „Stinkerstoffen" war in den Düften jedoch auch noch eine sehr große Bandbreite an Fettsäureestern (z. B. Ethyl- und Methylhexanoat, Butyl- und

Abb. 5 (oben): Absaugen des Blütenduftes. Hierzu wird die Blüte in einen Bratschlauch eingepackt, der Duft darin angereichert und dann über eine batteriebetriebene Pumpe über einen kleinen Filter, an dem die Duftstoffe adsorbieren, abgesaugt.

Abb. 6 (Seite 57): Labellum von Orchidantha fimbriata mit den charakteristischen Schleimabsonderungen. 


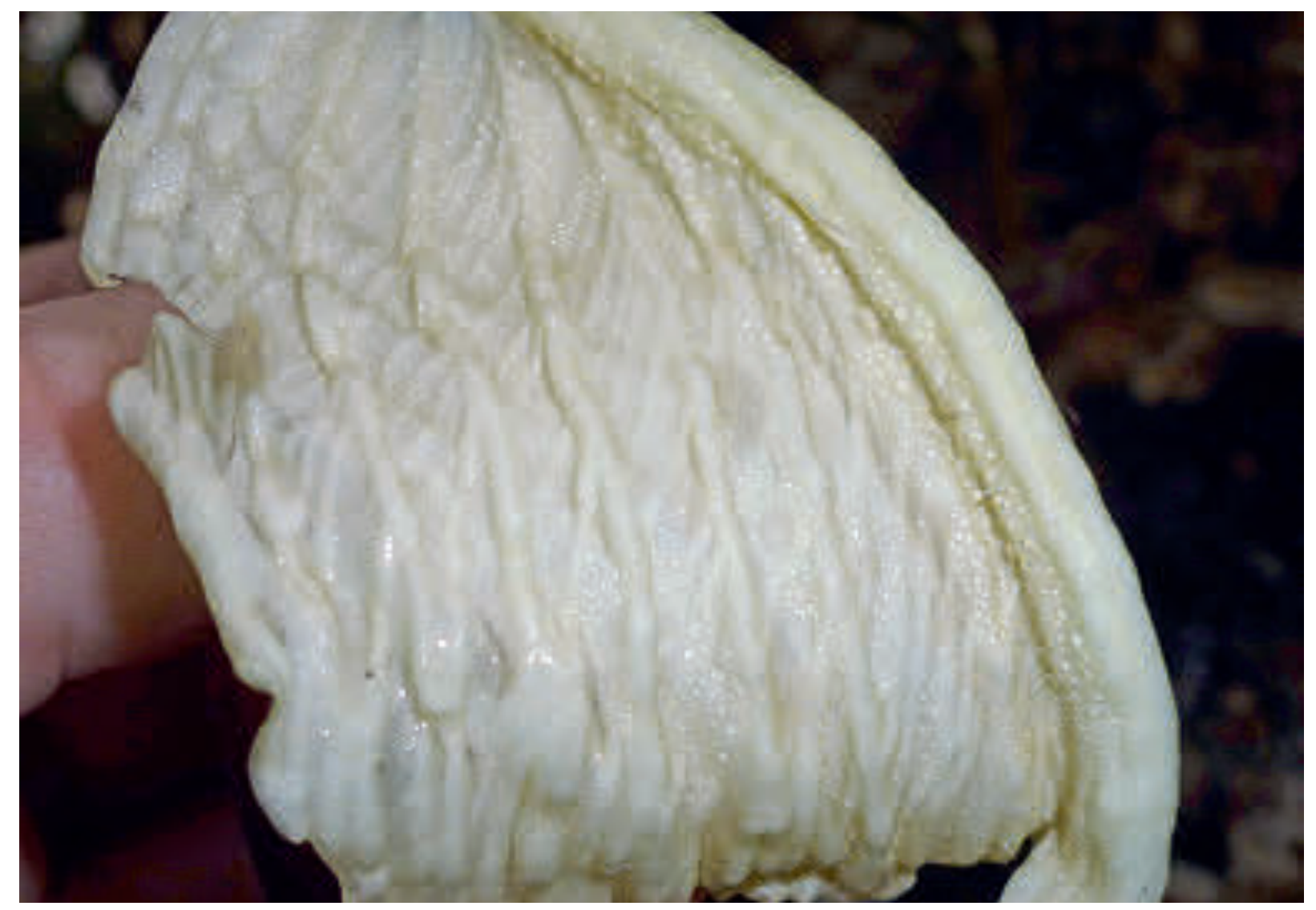

Ethylbuturat) enthalten. Auch diese sind bereits von anderen Kot oder Aas nachahmenden Pflanzen bekannt (Jürgens et al. 2013, Schiestl \& Dötterl 2012), sind jedoch nicht zu den "Stinkern“ zu zählen. Sie duften zum Teil fruchtig (Ethylbutyrat zum Beispiel nach Ananas), wobei diese fruchtige Komponente beim Riechen an Orchidantha fimbriata nicht oder kaum wahrnehmbar war.

Von den sogenannten "Stinkerstoffen“ ist bekannt, dass sie als Lockmittel für Insekten dienen, die Kot oder Aas als Brutsubstrat oder Nahrung nutzen (Jürgens et al. 2006). Von einigen Fruchtestern, die im Duft von O. fimbriata vorkommen, weiß man, dass sie Fruchtkäfer aus der Gruppe der Cetoniinae anlocken; so ist z.B. Ethylbutyrat ein sehr effizientes Lockmittel für afrikanische Fruchtkäfer der Art Pachnoda marginata (LARsson et al. 2003).

Diese Art von Substanzen könnte daher für die Anlockung der bestäubenden Fruchtkäfer bei Orchidantha fimbriata von Bedeutung sein, wie sie bei Petersen \& Johansen (2004) dokumentiert ist.
Wären aber Fruchtkäfer die einzigen Bestäuber von Orchidantha fimbriata, bliebe unklar, warum auch noch Kot- und Aas-typische Duftstoffe produziert werden. Diese würden auf eine Bestäubung durch Dungkäfer hindeuten, wie sie bei Orchidantha inouei nachgewiesen wurde (SAKAi \& InOue 1999), bei O. fimbriata aber bislang noch nicht dokumentiert wurde. Es muss daher offen bleiben, ob die Pflanze beide Käfergruppen als Bestäuber anlockt und nutzt. Denkbar wäre auch, dass die nachgewiesenen Kot- und Aasdüfte eine Funktion bei der Abschreckung von Blüten fressenden Organismen haben (Lev-YADUN et al. 2009), oder ein phylogenetisches „Überbleibsel“ darstellen.

Zusätzlich zu den bisher beschriebenen Substanzen wurden noch 23 verschiedene Sesquiterpene im Duft von $O$. fimbriata nachgewiesen. Diese Stoffgruppe tritt oft im Bouquet von Blütendüften auf, aber über ihre Funktion bei der Anlockung von Bestäubern ist bislang wenig bekannt (JüRGENS et al. 2006).

Eine weitere interessante Beobachtung war die Absonderung von klarem, glänzendem Se- 
kret auf dem gesamten Labellum von $O$. fimbriata (vgl. Abb. 6). Sekretabsonderung tritt z.B. auch bei Amorphophallus titanum auf und sorgt hier in der letzten Blühphase als Träger von nach verfaultem Fisch riechenden, stickstoffhaltigen Substanzen für eine weitere Intensivierung der Duftanlockung (Shirasu et al. 2010). $\mathrm{Ob}$ dieses Sekret bei O. fimbriata ebenfalls Träger von Duftstoffen und von welchen ist, müsste noch genauer untersucht werden.

Jedenfalls gehörte die Blüte von Orchidantha fimbriata im Dezember 2014 im ÖkologischBotanischen Garten der Universität Bayreuth zu den „Duft-“, aber auch zu den optischen Highlights im Gartenjahr.

\section{Dank}

Unser Dank gilt Frau Irmgard Schäffler für die Durchführung der GaschromatographieMassenspektrometrie.

\section{Literatur}

DAFNI, A. 1984: Mimicry and deception in pollination. Annu. Rev. Ecol. Syst. 15: 259-278.

Faegri, K. \& Pijl, L. van der 1979: The principles of pollination ecology. Third revised edition. - Oxford u.a.

Frost, S. K. \& Frost, P. G. H ...": Sunbird pollination of Strelitzia nicolai. - Oecologia 49: 379-384.

Holtтum, R. E. 1970: The genus Orchidantha (Lowiaceae). Garden's Bulletin SXXV: 239-247.

Jenjittikul, T. \& Larsen, K. 2002: Orchidantha foetida (Lowiaceae) a new species from Thailand. Nord. J. Bot. 22: 405-408.

Johansen, L. B. 2005: Phylogeny of Orchidantha (Lowiaceae) and the Zingiberales based on six DNA Regions. Sys. Botany 30: 106-117.

Jürgens, A., Dötterl, S. \& Meve, U. 2006: The chemical nature of fetid floral odours in stapeliads (Apocynaceae-Asclepiadoideae-Ceropegieae). New Phytol. 172: $452-68$.

Kite, G., Hetterscheid, W., Lewis, M., Boyce, P., Ollerton, J., Cocklin, E., Diaz, A. \&, Simmonds, M. 1998: Inflorescence odours and pollinators of Arum and Amorphophallus (Araceae). In: Owens S. and Rudall P. (eds.): Reproductive biology in systematics, conservation, and economic botany. - Kew.

Kress, W. J. 1990: The phylogeny and classification of the Zingiberales. - Ann. Missouri Bot. Gard. 77: 698-721. LARSEN, K. 1961: A new species of Veratrum and Orchidantha from Thailand and Laos. Sven. Bot. Tidskr. 56: $345-350$.
LARSEN, K. 1973: A new species of Orchidantha (Lowiaceae) from Vietnam. - Adansonia, ser. 2, 13: $481-482$.

LARSEN, K. 1993: A new species of Orchidantha (Lowiaceae) from Borneo. Nord. J. Bot. 13: 285-288. Larsson, M. C., Stensmyr, M. C., Bice S. B. \& Hansson, B. S. 2003: Attractiveness of fruit and flower odorants detected by olfactory receptor neurons in the fruit chafer Pachnoda marginata. - J. Chem. Ecol. 29: 1253-1268.

Lev-Yadun, S., Ne’eman, G. \& Shanas, U. 2009: A sheep in wolf's clothing: do carrion and dung odours of flowers not only attract pollinators but also deter herbivores? Bioessays 31: 84-88.

Nagamasu, H. \& SaKaI, S. 1999: Orchidantha inouei (Lowiaceae), a new species from Borneo. Nord. J. Bot. 19: 149-152.

Pedersen, L. B. 2001: Four new species of Orchidantha (Lowiaceae) from Sabah. - Nord. J. Bot. 21: 121-128.

Pedersen, L. B. \& Johansen B. 2004: Anatomy of the unusual stigma in Orchidantha (Lowiaceae). - Am. J. Bot. 91: 299-305.

Punekar, S. A. \& Kumaran, K. P. N. 2009: Pollen morphology and pollination ecology of Amorphophallus species from north western Ghats and Konkan region of India. Flora 205: 326-336.

Renner, S. S. 2006: Rewardless flowers in the angiosperms and the role of insect cognition in their evolution. In: Waser N. M. \& Ollerton J. (eds): Plant-pollinator interactions: From specialization to generalization. Chicago.

SAKaI, S. \& Inoue, T. 1999: A new pollination system: Dung-beetle pollination discovered in Orchidantha inouei (Lowiaceae, Zingiberales) in Sarawak, Malaysia. - Am. J. Bot. 86: 56-61.

Sakai S., Kato, M. \& Inoue, T. 1999: Three pollination guilds and variation in floral characteristics of Bornean gingers (Zingiberaceae and Costaceae). - Am. J. Bot. 86: 646-658.

Schiestl, F. P. \& Dötterl, S. 2012: The evolution of floral scent and olfactory preferences in pollinators: Coevolution or pre-existing bias? - Evolution 66: 2042-2055.

Shirasu, M., Fujioka, K., Kakishima, S., Nagai, S., Tomizawa, Y., Tsukay, H., Murata, J., Manome, Y. \& Tounara, K. 20Io: Chemical identity of a rotting animal-like odor emitted from the inflorescence of the titan arum (Amorphophallus titanum). - Biosci. Biotechnol. Biochem. 74: 2550-2554.

Stevens, P. F. 2001 onwards: Angiosperm phylogeny website. Version 12, http://www.mobot.org/MOBOT/ research/APweb/, abgerufen am 29.3.2015.

Stiles, F. G. 1975: Ecology, flowering phenology, and hummingbird pollination of some Costa Rican Heliconia species. - Ecology 56: 285-301. 